\title{
Searching for RF-Only Triggered Cosmic Ray Events with the High-Elevation BEACON Prototype
}

\author{
Daniel Southall ${ }^{1, *}$ on behalf of the BEACON Collaboration \\ (a complete list of authors can be found at the end of the proceedings) \\ ${ }^{1}$ Department of Physics, Enrico Fermi Institute, Kavli Institute for Cosmological Physics, \\ University of Chicago, 5720 S Ellis Ave \#201, Chicago, IL 60637, USA \\ E-mail: dsouthal1@uchicago.edu
}

The Beamforming Elevated Array for COsmic Neutrinos (BEACON) is a concept for a neutrino telescope designed to measure tau lepton air showers generated from tau neutrino interactions near the horizon. This detection mechanism provides a pure measurement of the tau flavor of cosmogenic neutrinos, which could be used to set limits on the observed flavor ratios for cosmogenic neutrinos in a manner complimentary to the all-flavor neutrino flux measurements made by other experiments. BEACON is expected to also be capable of detecting cosmic rays through RF-only triggers. BEACON aims to achieve this sensitivity by using mountaintop radio arrays of dual-polarized antennas operating in the $30-80 \mathrm{MHz}$ band which utilize directional interferometric triggering. BEACON stations are designed to efficiently use a small amount of instrumentation, allowing for deployment in a variety of high-elevation sites. The interferometric trigger provides a natural tool for directional-based anthropogenic RFI rejection at the trigger level, broadening the list for potential station sites. The BEACON prototype has seen continuous design advancements towards improving the mechanical durability and scientific capabilities since its initial deployment at White Mountain Research Station in 2018. Here we present the current prototype's sensitivity to RF-triggered cosmic-ray background signals. We also present the next generation prototype, which includes scintillating cosmic ray detectors, improved antennas, and refined calibration techniques.

This work is supported by NSF Awards \# 2033500, 1752922, 1607555, \& DGE-1746045 as well as the Sloan Foundation, the RSCA, and NASA (support through JPL and Caltech as well as Award \# 80NSSC18K0231). This work has received financial support from Xunta de Galicia (Centro singular de investigación de Galicia accreditation 2019-2022), by European Union ERDF, by the “María de Maeztu” Units of Excellence program MDM-2016-0692, the Spanish Research State Agency and from Ministerio de Ciencia e Innovación PID2019-105544GB-I00 and RED2018102661-T(RENATA). We thank the NSF-funded White Mountain Research Station for their support.

$37^{\text {th }}$ International Cosmic Ray Conference (ICRC 2021)

July 12 th - 23rd, 2021

Online - Berlin, Germany

\footnotetext{
*Presenter
} 


\section{Motivation}

Neutrinos from cosmic sources have been observed up to energies of several PeV[1] and are expected to have a significant flux at the EeV scale. Astrophysical neutrinos are expected to be produced at the sources, while cosmogenic neutrinos are expected to be produced through interactions of the highest energy cosmic rays with the cosmic microwave background. Energies above $100 \mathrm{PeV}$ are compelling for study, as in this range the expected cosmogenic neutrino flux [2] overlaps with extensions to the observed astrophysical flux [3-5]. Because only $v_{e}$ and $v_{\mu}$ are expected to be produced at the sources, an exclusive measurement of the $v_{\tau}$ flavor would essentially filter out any atmospheric production channels for neutrinos, and yield both flux and flavor ratio information for testing both cosmogenic and astrophysical neutrino models.

The Beamforming Elevated Array for COsmic Neutrinos (BEACON) concept consists of mountaintop phased radio antennas that are designed for measuring the flux of astrophysical $v_{\tau}$ above $100 \mathrm{PeV}$ [6]. As a $v_{\tau}$ interacts with the surface of the Earth it can produce a $\tau$ lepton, which can escape the Earth's crust and decay in the atmosphere. This decay creates an upgoing extensive air shower that will produce an impulsive radio pulse via a combination of the geomagnetic radiation and Askaryan radiation [7]. As it is based on 100s to 1000s of independent stations, the BEACON concept would utilize a global network of low-cost high-elevation mountaintop radio arrays to search for these signals. The high elevation provides the optimal field of view for horizon-based searches, while a noise-riding phased directional trigger provides anthroprogenic Radio Frequency Interference (RFI) rejection, while maintaining sensitivity to the expected diffuse flux.

A BEACON prototype has seen ongoing development and support since 2018 [8]. These proceedings discuss all of BEACON, with significant emphasis on providing updated details on the existing prototype, plans for the future, and the cosmic ray analysis being performed on data from the prototype.

\section{The BEACON Prototype}

The BEACON prototype is located near Barcroft Field Station, in the White Mountains near Bishop, California, USA. The first installation in 2018 used crossed-V dipoles [8]; however, since 2019 the prototype has consisted of 4 crossed electrically-short-dipole antennas, each driven by an active feed and nominally sensitive in the $30-80 \mathrm{MHz}$ range. The design of these custom antennas has seen improvements annually, with both high-impedance and balun transformer matching circuits being tested (with galactic noise visible in at least the VPol antennas as shown in Figure 2). The current antennas are elevated $\sim 3.7 \mathrm{~m}(12 \mathrm{ft})$ above the mountainside via masts constructed of both wood (for low-interference structural support near the antenna) and steel signposts (for structural support and grounding). In order to withstand the extreme weather at the prototype site, the masts are secured by $\geq 6$ guy-lines, 4 wooden struts, and a $37 \mathrm{~kg}(70 \mathrm{lb})$ rubber base each. This design has proven to be capable of deploying stable masts in locations of extremely irregular terrain, where drilling or pouring cement is not an option, and is flexible enough to support different terrains.

The prototype utilizes a phased trigger system modeled after the ARA 5 phased array [9]. This trigger has a field programmable gate array (FPGA) which utilizes a pre-calculated table of expected arrival time differences between the antennas to delay signals before performing a power sum on 

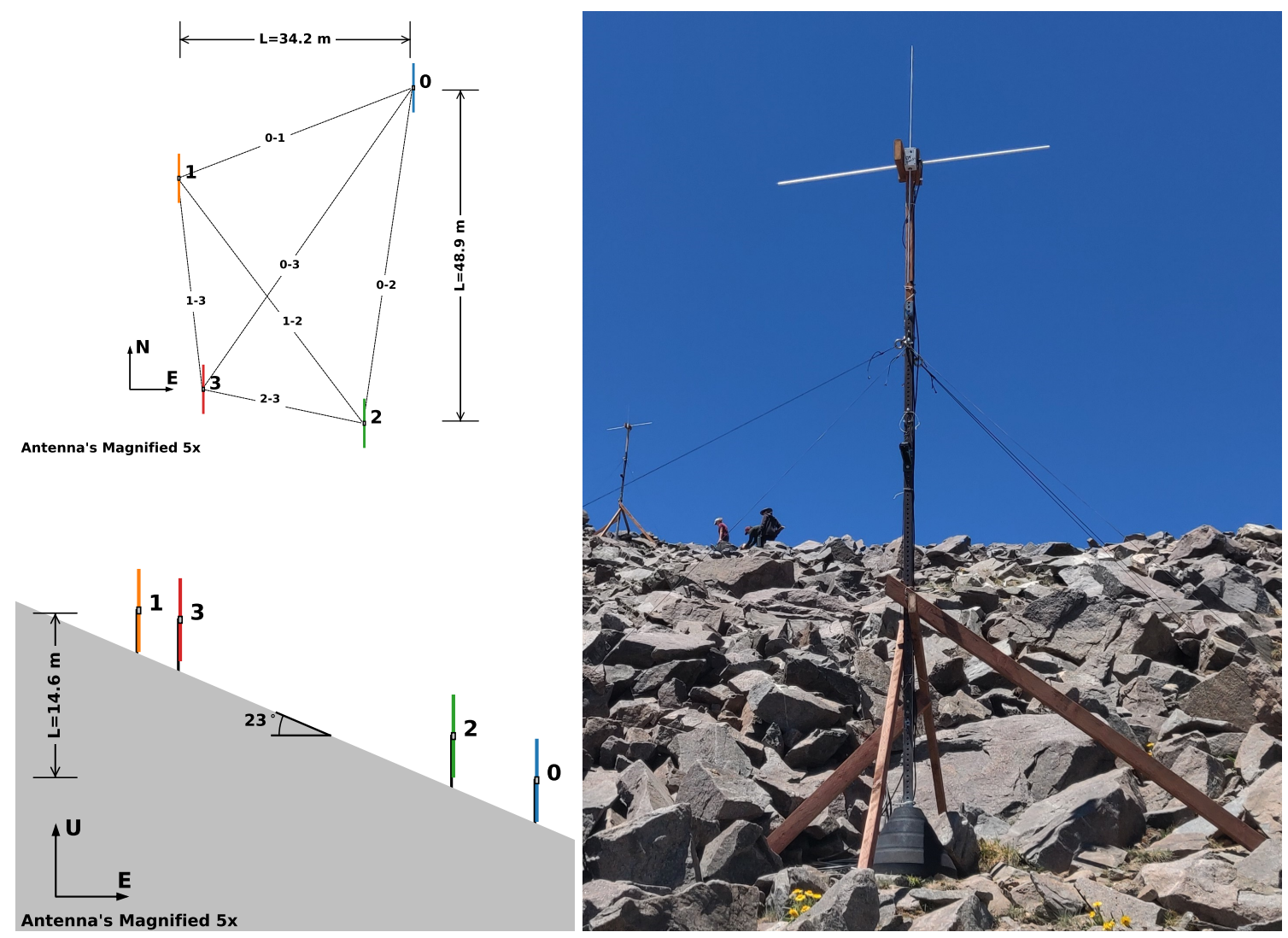

Figure 1: Upper Left: A top-down view of the array in East-North-Up (ENU). Lower Left: A side profile of the array showing the elevation differences in the antennas. Right: BEACON mast 0 with crossed-dipole antennas, rubber base, wooden struts, and guy-lines. The displayed position measurements were taken with an RTK-GPS system in June 2021, with mast photograph taken during the same time.
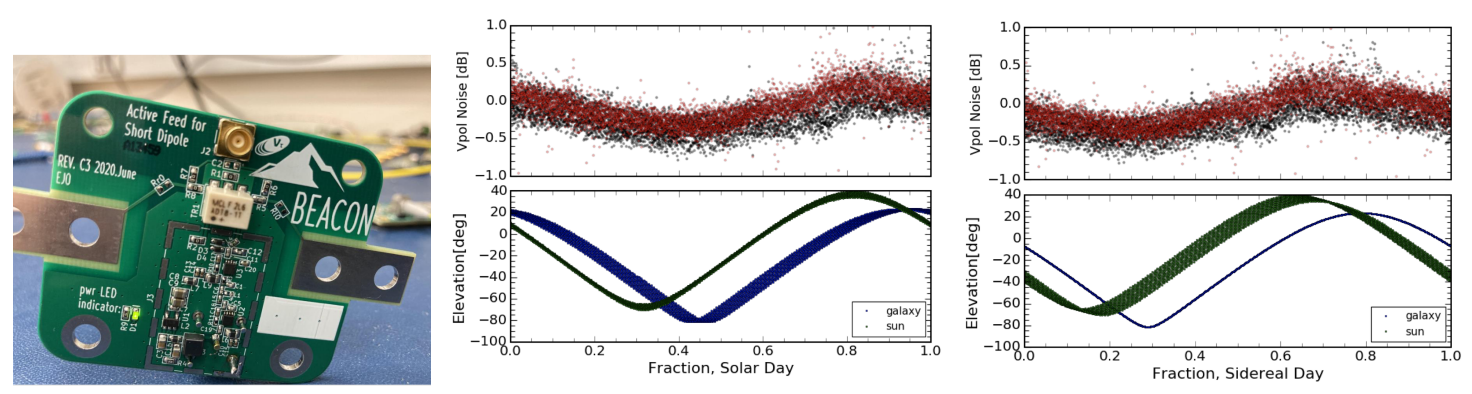

Figure 2: Left: The custom-designed active feed for the BEACON antennas. Right: The root-mean-squared noise measured on the two of the electrically short vertically polarized antennas used in the BEACON prototype in 2020 compared with the elevation of the sun (green) and the galactic center (blue). While the RMS noise is expected to vary with temperature, which is driven by solar heating, the galactic center appears visible to these antennas. 
the resulting net signal. Each set of time delays corresponds to a particular direction, referred to as a "beam", and is sensitive to the specific direction where its delays result in coherently summed signals. This coherent sum will increase the signal's magnitude by a factor of $N_{\text {antenna, while the }}$ noise will add incoherently and only increase as $\sqrt{N_{\text {antenna }}}$, resulting in a net signal-to-noise ratio (SNR) increase of $\sqrt{N_{\text {antenna }}}$ [9].

The current analysis goal of the BEACON prototype is to prove the capability to RF-trigger on cosmic rays. In a full-scale BEACON, cosmic rays will be a source of background, sharing many characteristics with the expected $v_{\tau}$ signals, but distinguishable based on direction and polarity. Observing cosmic rays in the prototype will provide both a proof of concept and validation of the system. The observed rate and threshold will determine the detector trigger threshold and set the expected sensitivity for a full-scale BEACON. Detector validation and the search for cosmic rays is ongoing.

\section{Position Calibration}

\subsection{Calibration Background}

Accurate source direction reconstruction (both at the trigger level and in analysis) requires precise knowledge of the array timing, including the location of each antenna and signal cable lengths. A calibrated array can use pointing for RFI rejection of static or transient sources like settlements or airplanes respectively, as well as for characterization of the polarization and source properties of the initiating radio source. The typical method of calibrating an array consists of the following process: 1. Perform initial position measurements using a Global Positioning System (GPS) system or similar; 2. Measure cable delays using a vector network analyzer or time-domain reflectometer (TDR); 3. Send radio pulses at the array from a known location (also measured with comparable system to antennas), recording the pulses through the data acquisition system (DAQ) for later analysis; 4. Perform a $\chi^{2}$ minimization (with antenna positions being the dynamic parameters) of the residual of predicted arrival time differences in each channel against the actual measured delays through the DAQ.

For the BEACON prototype the number of degrees of freedom for the minimization parameters is equal to 3 axis of movement +1 cable delay per antenna, leading to 16 total degrees of freedom (DoF). The number of distinct measurements provided by $N_{\text {site }}$ pulsing sites is given by DoF = $N_{\text {site }} \cdot C\left(N_{\text {antenna }}, 2\right)$ where $C(n, r)$ is the choose operator, which determines the number of arrival time differences that can be calculated when comparing 2 antennas from a set of $N_{\text {antenna. }}$.

The number of pulsing sites is highly constrained on site due to the rough terrain and high elevation. For this reason, the theoretical minimum number of necessary pulsing sites was chosen during the 2019 prototype upgrade/deployment. A pulser was carried to 3 sites ranging from $\sim 350$ $\mathrm{m}$ to $\sim 1100 \mathrm{~m}$ meters covering an azimuthal span of $105^{\circ}$ and an elevation span of $3^{\circ}$ (which is constrained by the pulser residing on the mountainside). At each site a bicone pulser was triggered on the GPS second for 10-20 minutes; the position of each pulsing site was measured using the same GPS system. 


\subsection{The Calibration Effort}

Offline analysis of this data set began shortly after the October 2019 deployment, and uncovered many potential improvements that would lead to better constraints on the position calibration in the 2021 season. The GPS system that measured the pulsing sites was not used to measure the initial positions of the receiving antennas on the mountainside, and a combination of mismatching accuracy and elevation standards resulted in uncertainty that lead to the pulsing site locations being treated as unknowns in early calibration efforts - which adds 3 DoF per pulsing site, under-constraining the problem. This could be remedied in a follow-up deployment, however due to the seasonal access to the prototype site and the global COVID-19 pandemic, access to the site for a follow-up deployment was not possible until June $2021-\sim 1.5$ years later (at which point they were resolved, as discussed below). In the meantime work was done to attempt a thorough calibration with the information available. Using initial position measurements and rough calibrations, candidates for sources of RFI were identified. If accurately attributed, each source of RFI could effectively increase $N_{\text {site }}$, and because these RFI sources were up-to 2 orders of magnitude further from the array than our pulsing sites - the effect of uncertainties in their positions were minimized.

The area visible to the BEACON prototype is largely empty California/Nevada desert, however RFI sources are still visible (see Figure 3). Ultimately there is ambiguity in visually linking signals to potential sources, so iterations of the minimization were also performed using a source-direction agnostic minimization metric that prioritized overlapping/consistent pointing from each observed arrival time difference pair - regardless of where that overlap occurs in the sky. This metric is effectively the same as letting the RFI source position move on the surface of a sphere - adding 2 unknowns, reducing each additional $N_{\text {site }}$ net ability to constrain from 6 to 4 degrees of freedom, while avoiding pitfalls such as forcing a calibration to match misidentified source directions.

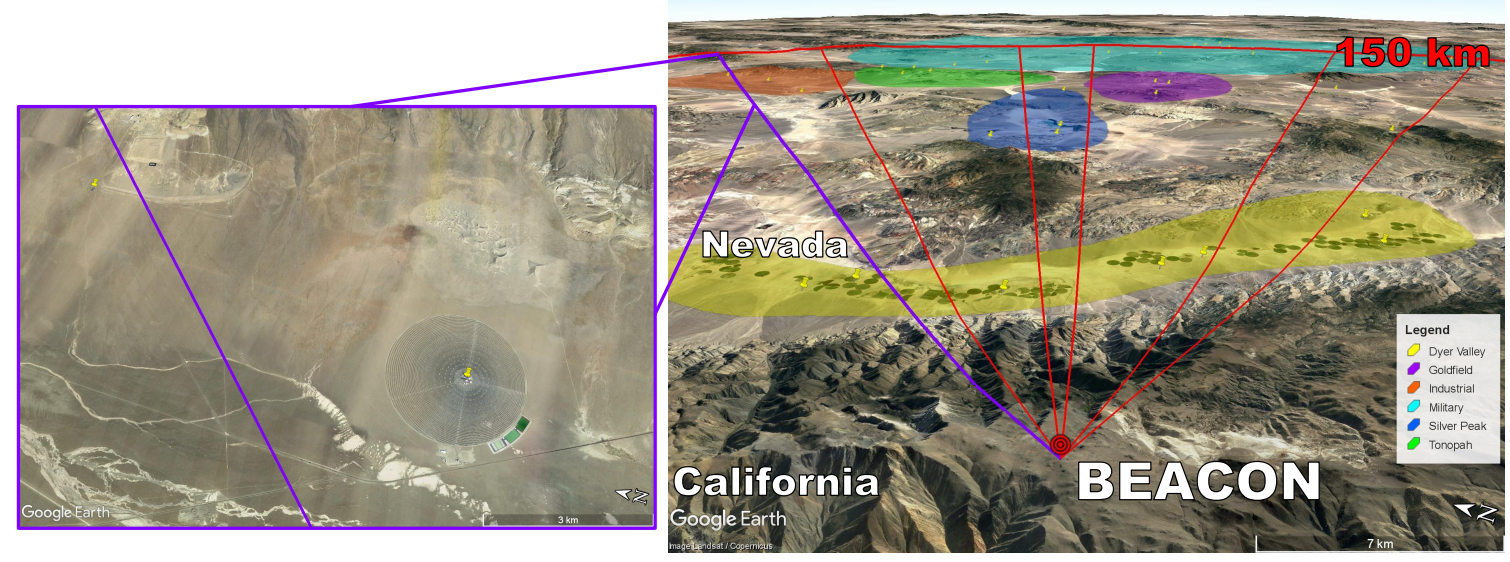

Figure 3: An aerial view map of the visible area from the BEACON prototype site, including directions with prominent RFI sources (rays), and general area descriptions. Small yellow pins indicate visually identified candidate RFI sources. The source highlighted by the purple ray corresponds to the highlighted clusters in Figure 4, and is believed to be related to the Crescent Dunes Solar Energy Project (visible in the pop-out image on the left).

The resulting calibration derived from the above technique was largely validated by follow-up GPS measurements made during the June 2021 deployment. These measurements utilized new 
permanently installed GPS patch antennas, which are now located on each mast. These antennas were connected to Ublox C099-F9P application boards to perform Real-time Kinematic (RTK) GPS measurements enhanced by Networked Transport of RTCM via Internet Protocol (NTRIP) data provided by a conveniently locally installed UNAVCO GPS station (permanently installed $\sim 30$ meters from our closest BEACON antenna). This corrective data accounts for fluctuations in the atmosphere by comparing GPS positioning of 2 close-by antennas - resulting in $\mathrm{cm}$ level precision. This system has been shown to work well and will be used for position measurements moving forward. We also plan to increase the ease of pulsing in the future via the use of an aerial drone pulser which is under development.

\section{Ongoing Cosmic Ray Search}

Determining the sensitivity of the BEACON prototype to cosmic rays (BEACON's main source of astrophysical background, and a source that is has very similar characteristics to a $v_{\tau}$ event) would provide a strong proof of concept, as well as enable us to refine the sensitivity estimates of a fullscale BEACON array. Triggering on such events has been shown to be possible [10, 11], however not with a small array and phased-trigger like that implemented with the BEACON prototype nor from a high-elevation mountain.

Moving forward we intend to use the achieved position calibration to analyze both the eligible data taken with the 2019 deployment, as well as incoming data from the new 2021 deployment. This search will implement generous and thorough RFI rejection using both direction reconstruction, spatial and temporal clustering, and signal property analysis. The upper plots of Figure 4 show high clustering in both the reconstruction direction and peak-to-peak (P2P) parameter phase spaces. The lower plots show that the angular resolution of each individual source is sub-degree scale. Clustering exists for all measured quantities, such as signal-to-noise ratio, arrival time delays, cosmic ray template searches, impulsivity, etc. Tools have been developed to easily cut on any $N$ dimensions of the available phase space of parameters, allowing for isolation of single RFI source as highlighted by the purple contours in these plots. Such analysis will also allow us to confidently remove many events that mis-reconstruct above the horizon due to prominent sidelobes. The clustering of these events also suggests that directional masking may be useful in rejecting RFI at the trigger level, which will be the subject of future study.

Once all identified RFI events have been removed, analysis of the observed polarization, event rates as a function of zenith angle, cosmic ray template quality comparisons, and other similar metrics will all be considered and compared to simulation before our final cosmic ray observation claims are made. Much of the framework for these cuts has already been developed alongside the lengthy calibration process. For information regarding the development of BEACON's cosmic ray simulation please see an accompanying proceeding [12].

\section{Conclusion}

While analysis of the data collected by the 2019 prototype is ongoing, improvements in calibration and mechanical design of the June 2021 BEACON prototype have already provided a promising (and expanding) data set. We have already demonstrated the ability of our directional 

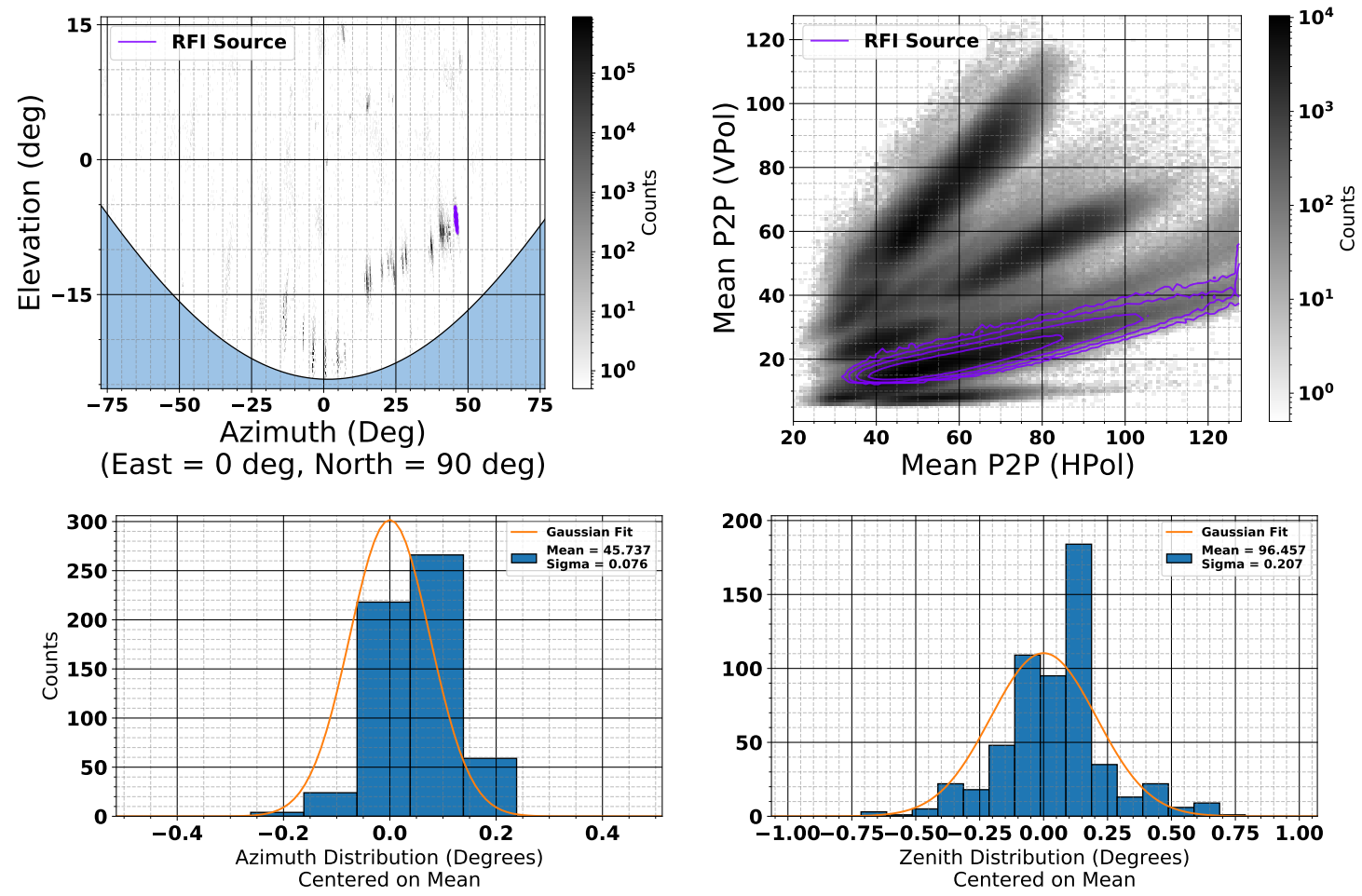

Figure 4: Upper Left: The reconstruction direction of RF triggered events from early runs in the 2019 deployment. The blue shading approximates the location and slope of the local mountainside. Clusters of events above $0^{\circ}$ elevation are typically events that have reconstructed to a side lobe of a below-horizon RFI source, and can be accounted for in analysis. Upper Right: The peak-to-peak (P2P) values of both horizontal and vertical antennas for each event, illustrating clear separation of signal properties for disparate RFI sources. Lower: The angular reconstruction distributions for $\sim 1$ hour of events from the highlighted purple RFI source. This RFI source can also be seen in Figure 3.

trigger for identifying and suppressing static RFI sources. With the calibration techniques developed thus far we are confident that we will be able to set a limit on the cosmic ray rate at our site, and thus provide solid insights into the capabilities of a full-scale BEACON array in the near future. Progress towards hardware/system upgrades such as the drone pulser will continue to be made to the BEACON prototype while this analysis is being performed. The addition of several scintillating particle detectors is planned for both future prototype enhancement and for the full-scale detector. With such an upgrade the system would still be RF-triggered, but with additional information from the scintillators serving as an easy discriminator between cosmic rays (which should produce signals in the detectors) and other sources of impulsive signals.

\section{References}

[1] M. Aartsen, R. Abbasi, M. Ackermann, J. Adams, J. Aguilar, M. Ahlers et al., Icecube-gen2: the window to the extreme universe, Journal of Physics G: Nuclear and Particle Physics $\mathbf{4 8}$ (2021) 060501. 
[2] K. Kotera and A.V. Olinto, The astrophysics of ultrahigh-energy cosmic rays, Annual Review of Astronomy and Astrophysics 49(1) (2011) 119.

[3] M. Aartsen, K. Abraham, M. Ackermann, J. Adams, J.A. Aguilar, M. Ahlers et al., A combined maximum-likelihood analysis of the high-energy astrophysical neutrino flux measured with icecube, The Astrophysical Journal 809 (2015) 98.

[4] M. Aartsen, K. Abraham, M. Ackermann, J. Adams, J. Aguilar, M. Ahlers et al., Observation and characterization of a cosmic muon neutrino flux from the northern hemisphere using six years of icecube data, The Astrophysical Journal 833 (2016) 3.

[5] M. Aartsen, M. Ackermann, J. Adams, J. Aguilar, M. Ahlers, M. Ahrens et al., Search for astrophysical sources of neutrinos using cascade events in icecube, The Astrophysical Journal 846 (2017) 136.

[6] S. Wissel, J. Alvarez-Muniz, C. Burch, A. Cummings, W. Carvalho, C. Deaconu et al., Concept study for the beamforming elevated array for cosmic neutrinos (beacon), in 36th International Cosmic Ray Conference, vol. 358, p. 1033, SISSA Medialab, 2019.

[7] J. Alvarez-Muniz, W.R. Carvalho Jr and E. Zas, Monte carlo simulations of radio pulses in atmospheric showers using zhaires, Astroparticle Physics 35 (2012) 325.

[8] K. Hughes, J. Alvarez-Muñiz, W. Carvalho Jr, A. Cummings, C. Deaconu, G. Hallinan et al., Towards interferometric triggering on air showers induced by tau neutrino interactions, PoS (ICRC2019) 917 (2019) .

[9] P. Allison, S. Archambault, R. Bard, J. Beatty, M. Beheler-Amass, D. Besson et al., Design and performance of an interferometric trigger array for radio detection of high-energy neutrinos, Nuclear Instruments and Methods in Physics Research Section A: Accelerators, Spectrometers, Detectors and Associated Equipment 930 (2019) 112.

[10] R. Monroe, A.R. Wolf, G. Hallinan, A. Nelles, M. Eastwood, M. Anderson et al., Self-triggered radio detection and identification of cosmic air showers with the ovro-lwa, Nuclear Instruments and Methods in Physics Research Section A: Accelerators, Spectrometers, Detectors and Associated Equipment 953 (2020) 163086.

[11] D. Charrier, K.D. de Vries, Q. Gou, J. Gu, H. Hu, Y. Huang et al., Autonomous radio detection of air showers with the trend50 antenna array, Astroparticle Physics 110 (2019) 15.

[12] A. Zeolla, S.A. Wissel, J.A.-M. niz, W.C. Jr., A. Cummings, Z. Curtis-Ginsberg et al., Modeling and validating rf-only interferometric triggering with cosmic rays for beacon, PoS (ICRC2021) (2021) . 


\section{Full Authors List: BEACON}

D. Southall ${ }^{1, *}$, S. A. Wissel ${ }^{2,3}$, J. Alvarez-Muñiz ${ }^{4}$, W. Carvalho $\mathrm{Jr}^{5}{ }^{5}$, A. Cummings ${ }^{6}$, Z. Curtis-Ginsberg ${ }^{1}$, C. Deaconu ${ }^{1}$, K. Hughes ${ }^{1}$, A. Ludwig ${ }^{1,7}$, K. Mulrey ${ }^{8}$, E. Oberla ${ }^{1}$, S. Prohira ${ }^{9}$, A. Romero-Wolf ${ }^{10}$, H. Schoorlemmer ${ }^{11}$, A. G. Vieregg ${ }^{1}$, E. Zas ${ }^{4}$, A. Zeolla ${ }^{2}$

${ }^{1}$ University of Chicago, ${ }^{2}$ Penn State University, ${ }^{3}$ California Polytechnic State University, ${ }^{4}$ Instituto Galego de Física de Altas Enerxías IGFAE, Universidade de Santiago de Compostela, 15782 Santiago de Compostela, Spain, ${ }^{5}$ Univerisade de São Paolo, Brazil, ${ }^{6}$ Gran Sasso Science Institute, ${ }^{7}$ University of California, Los Angeles, ${ }^{8}$ Physics Dept., Vrije Universiteit Brussel, ${ }^{9}$ The Ohio State University, ${ }^{10}$ Jet Propulsion Laboratory, California Institute of Technology, ${ }^{11}$ Max-Planck-Institut für Kernphysik 